\title{
HIV-associated lipodystrophy: a review from a Brazilian perspective
}

\author{
This article was published in the following Dove Press journal: \\ Therapeutics and Clinical Risk Management \\ 17 July 2014 \\ Number of times this article has been viewed
}

\section{Marcelle D Alves' \\ Carlos Brites ${ }^{2}$ \\ Eduardo Sprinz ${ }^{1,3}$}

'Infectious Disease Department, Hospital de Clínicas de Porto Alegre,

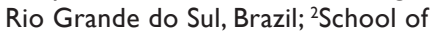
Medicine, Federal University of Bahia, Salvador, Brazil; ${ }^{3}$ School of Medicine, Federal University of Rio Grande do Sul, Porto Alegre, Brazil
Correspondence: Eduardo Sprinz Hospital de Clínicas de Porto Alegre, Rua Ramiro Barcelos 2350, Sala 700, Porto Alegre, RS 90035-003, Brazil Tel/Fax +55 5I 33598 II52

Email eduardo.sprinz@gmail.com
Abstract: The prognosis of human immunodeficiency virus (HIV)-infected individuals has dramatically improved worldwide since the introduction of highly antiretroviral therapy. Nevertheless, along with the decrease in mortality, several body modifications not initially related to HIV infection have been reported. Disorders in lipid and glucose metabolism, accompanied by body shape abnormalities and alterations in fat distribution, began to be described. A syndrome, named "HIV-associated lipodystrophy syndrome", was coined to classify these clinical spectrum aspects. This syndrome involves not only metabolic alterations but also fat redistribution, with lipoatrophy due to subcutaneous fat loss (predominantly in the face and lower limbs) and lipohypertrophy related to central fat gain. These changes in body shape are very important to be recognized, as they are associated with worse morbidity and mortality. Selfesteem difficulties related to body alterations might lead to treatment failures due to medication adherence problems. Moreover, these alterations have been associated with an increased risk of cardiovascular events. Therefore, it is extremely important to identify this syndrome early in order to provide an even better quality of life for this population, as the clinical approach is not easy. Treatment change, medications to treat dyslipidemia, and surgical intervention are instruments to be used to try to correct these abnormalities. The aim of this study is to review clinical presentation, diagnosis, and management of body shape and metabolic complications of HIV infection from a Brazilian perspective, a medium income country with a large number of patients on antiretroviral therapy.

Keywords: lipoatrophy, lipodystrophy, lipohypertrophy, HAART, dyslipidemia, Brazil

\section{Introduction}

The prognosis of human immunodeficiency virus (HIV)-infected individuals has improved significantly since the introduction of highly active antiretroviral therapy (HAART), and mortality rates have dramatically diminished worldwide. ${ }^{1-3}$ Brazil has more than 700,000 people infected with HIV, and the number of patients on antiretroviral therapy (ART) in the country has doubled in the last 10 years. Nowadays, over 300,000 patients are on HAART, and about $70 \%$ of them have viral loads below the level of detection. ${ }^{4}$ Nevertheless, as these individuals are living longer, several complications are increasingly being reported, some associated with longer survival and some related to $\mathrm{ART}^{5}$

Disturbances in lipid and glucose metabolism, along with body shape abnormalities and alterations in fat distribution, were one of the first described, mostly occurring as a consequence of ART. A syndrome was coined to classify these clinical aspects, "HIV-associated lipodystrophy syndrome", ${ }^{6}$ although the current approach 
is to consider the components as different entities. ${ }^{7,8}$ These body alterations are usually associated with a decrease in self-esteem, which might lead to problems with adherence to ART and ultimately to treatment failure. This syndrome, no matter which components are manifested, is associated with an increased risk for cardiovascular disease (as a consequence of metabolic alterations, mainly dyslipidemia, and increased peripheral resistance to insulin). Lipoatrophy, lipohypertrophy, and fat redistribution have already been described in Brazilian patients infected by HIV. ${ }^{9-11}$ The aim of this article is to review clinical presentation, diagnosis, and management of body shape abnormalities in HIV infection from a Brazilian perspective, a medium income country with a large number of patients on ART.

\section{HIV-associated lipodystrophy syndrome}

HIV-associated lipodystrophy was initially described as a combination of fat loss in limbs and central fat accumulation. ${ }^{6}$ These shape abnormalities were first described as a syndrome in 1998 and included lipoatrophy, lipohypertrophy, and metabolic disturbances. Over the last two decades, some investigations have led to a better understanding of these abnormalities. It became clear that there is no single morphologic syndrome and that it is more valid to consider its individual components separately, as the pathogenesis of each may differ. ${ }^{7,8,11}$

Lipoatrophy is related to subcutaneous fat loss. It typically involves limbs (predominance of lower limbs), the face, and buttocks. This phenomenon has been extensively related to nucleoside reverse transcriptase inhibitor (NRTI) use, mainly thymidinic analogs (greater with stavudine [d4T] and less with zidovudine [ZDV]). It is likely to be an adverse event related to NRTI-induced inhibition of mitochondrial deoxyribonucleic acid polymerase $\gamma{ }^{11,12}$ Although some studies initially suggested that the use of protease inhibitors (PIs) was associated with lipoatrophy, now it seems more clear that this class of drugs is not responsible, as treatment regimens based exclusively on PIs were not found to be related to this abnormality. ${ }^{13,14}$ This has been elegantly demonstrated by the AIDS Clinical Trials Group (ACTG) 5142 study, in which the incidence of lipoatrophy was significantly higher in the efavirenz plus two NRTIs group than in the lopinavir plus two NRTIs or efavirenz plus lopinavir groups (32\% versus $17 \%$ versus $9 \%$, respectively). The association of lipoatrophy with efavirenz use was mainly in combination with either $\mathrm{d} 4 \mathrm{~T}$ or ZDV but not with tenofovir plus lamivudine. ${ }^{14}$

On the other hand, lipohypertrophy consists of the accumulation of adipose tissue. It normally involves the intra-abdominal (increased visceral adipose tissue), breast, anterior neck, and dorsocervical ("buffalo hump") regions. ${ }^{8,11}$ Although a role for PIs in the development of lipohypertrophy has been unmistakably described since the beginning of their use, ${ }^{15-17}$ some longitudinal studies have failed to demonstrate this association. ${ }^{7,18-20}$ A systematic review that included 27 studies of lipoatrophy (but not lipohypertrophy) was associated with ART. Central fat gain was not different from the controls and reflected a pattern of fat gain seen in the HIV-uninfected population. ${ }^{13}$

The other alteration included in the syndrome is dyslipidemia, which can occur along with lipoatrophy and/or lipohypertrophy, although it can be present in the absence of abnormalities of body shape. Most PIs, with the exception of atazanavir and darunavir, are associated with an elevation in levels of total cholesterol, low-density cholesterol, and triglycerides. ${ }^{21,22}$ PIs have a direct effect on lipid metabolism. Several mechanisms, including molecular homology between HIV protease and some proteins involved in lipid metabolism and inhibition of plasmatic lipoprotein lipase, have been described. ${ }^{11,23}$ Genetic predisposition could explain, at least in part, the large heterogeneity between patients in relation to the intensity of the effects of PIs on lipid metabolism. ${ }^{24}$ Elevations in total cholesterol can also occur with d4T, ZDV, and efavirenz. ${ }^{25}$

Likely, there are other aspects that could justify or even help the development of lipodystrophy. Some studies have demonstrated that obesity produces a milieu for inflammatory proteins, and inflammation as immune activation may play a role in HIV-associated lipodystrophy. In well-controlled patients (on ART and undetectable viral load), CD8+ T-cell activation may play a potential role in lipodystrophy and visceral fat accumulation. ${ }^{26}$

Disruptions in glucose metabolism are part of the metabolic effects of antiretroviral drugs, and a spectrum of disorders of glucose metabolism has been associated with HIV infection and ART. PIs (again, perhaps with the exception of atazanavir and darunavir) inhibit glucose transporter type 4 in the transport of glucose and can alter the adipocyte differentiation, contributing to the development of fat redistribution and insulin resistance. ${ }^{11,21}$ NRTIs (mainly d4T and, less so, ZDV) can also be associated with dyslipidemia and insulin resistance due to mitochondrial toxicity. ${ }^{11,21,23}$

Some conditions have been implicated in lipodystrophy. Loss of subcutaneous fat, lower CD4 cell count, and higher baseline viral load were predictors of lipoatrophy in some studies. ${ }^{21}$ Increased age has been associated with self-reported lipoatrophy, although age was not linked to subcutaneous fat 
loss when using a dual energy X-ray absorptiometry (DEXA) scan. ${ }^{27}$ The risk of developing lipoatrophy has been shown to be increased in patients with low baseline tricep skin fold values and smaller hips, according to Jacobson et al. ${ }^{28}$ Hepatitis C coinfection has been described as a feature associated with HIV lipoatrophy. ${ }^{21}$

Regarding sex, central fat accumulation is more frequent in females and is associated with increased body fat. ${ }^{28}$ Studies that have examined the quality of diet in HIV-infected individuals with and without visceral adiposity suggest that poor diet quality, as measured by amount of fiber ingested, is associated with the occurrence of fat deposition. ${ }^{29}$

All these metabolic abnormalities described here are associated with an increased risk of cardiovascular risk in the general population. Likewise, a number of studies have also shown that there is an increased risk of cardiovascular disease in HIV-infected versus uninfected populations, mainly in patients presenting these modifications. ${ }^{30-33}$

\section{Epidemiology}

The absence of an objective case definition of HIV-associated lipodystrophy has led to substantial variations in reports of prevalence, incidence, severity, risk factors, and responses. Lipodystrophy prevalence varies between $10 \%$ and $80 \%$. This wide variation may also be related to differences in geographic area, age, genetics, and lifestyle factors, as well as methodological differences between the studies. ${ }^{28,34-36}$

Longitudinal studies that evaluated fat atrophy have described an incidence of around $35 \%{ }^{28,35}$ along with a $13 \%$ median loss of limb fat per year. ${ }^{37}$ Subcutaneous fat atrophy is more common in HIV-positive individuals when compared with noninfected individuals. ${ }^{6,38}$ The prevalence of fat accumulation has been described at around $40 \%{ }^{28,35}$ Appropriate data in Brazil are limited to very few studies. In a cross-sectional study performed in Sao Paulo, 64\% of 457 patients evaluated self-reported body shape abnormalities; 49\% reported fat accumulation and $37 \%$ reported subcutaneous fat loss. ${ }^{9}$ Studies from other parts of the country showed similar data. ${ }^{10,39}$

Today, according to nutritional status, obesity and overweight are found more often than malnutrition. This is in contrast to the beginning of the epidemic, when full-blown acquired immunodeficiency syndrome (AIDS) was associated with caquexia and severe malnutrition. There has been an important change over the past years, which parallels the progress of ART and the control of HIV infection. ${ }^{40}$ According to Lazzaretti et al, ${ }^{41}$ the risk of an HIV-positive individual who starts ART to develop dyslipidemia in Brazil can be as high as $70 \%$.

\section{Diagnosis}

The diagnosis of lipodystrophy includes anthropometric evaluation and physical and radiologic examination. Anthropometric evaluation includes measurements of weight, height, body mass index, skin folds, and circumferences (waist, hip, and limbs). These measurements help to identify alterations in body shape and in body composition but are not specific to lipoatrophy or lipohypertrophy. ${ }^{21}$

Tricep skin fold less than tenth percentile according to the National Health and Nutrition Examination Survey for sex and age has been used in studies to define lipoatrophy. ${ }^{28}$ Abdominal circumference $(>102 \mathrm{~cm}$ for men and $>88 \mathrm{~cm}$ for women) and a waist/hip ratio ( $>0.95$ in men and $>0.85$ in women) can be used to screen abdominal obesity; however, they do not distinguish between visceral and subcutaneous adipose tissue accumulation. ${ }^{21}$

Quantification of fat can be obtained by computed tomography (CT), magnetic nuclear resonance (MNR), and DEXA scan. However, they are expensive and require expert interpretation, making their use limited to research. $^{21}$

The major difference between lipoatrophy and AIDSassociated wasting is the preferential loss of subcutaneous adipose tissue without loss of lean mass. In patients with lipoatrophy, loss of subcutaneous fat in limbs can be evident as musculature and veins become more prominent. Truncal lipoatrophy can be less noticeable, especially when visceral fat deposition occurs concomitantly; in this situation, abdominal circumference and waist/hip ratio may not reveal the change. ${ }^{21}$

Facial lipoatrophy is of special concern due to its facial wasting appearance and the impact on self-esteem. There are no objective techniques widely accepted to evaluate facial lipoatrophy; some staging scales have been proposed. ${ }^{21}$ Measurement of facial atrophy by ultrasonography has a good sensitivity, but its use is limited to research. ${ }^{42}$

The measurement of abdominal circumference and waist/hip ratio are useful diagnostic procedures to evaluate abdominal obesity. Sometimes it is difficult to differentiate lipohypertrophy from simple obesity. Although CT and MNR can be used as objective measurements of visceral fat, a DEXA scan cannot distinguish between visceral and subcutaneous adipose tissue, thus cannot be used in the screening of lipohypertrophy. ${ }^{12}$

Although physical examination and anthropometric evaluation are used to diagnosis lipoatrophy and lipohypertrophy, patient self-report can be an early and valuable indicator of body changes. ${ }^{12}$ Several Brazilian studies have used 
anthropometric evaluation (which is cheaper) and patient self-report to diagnose body fat redistribution. $9,10,40,43$

\section{Treatment}

The treatment approach depends on the nature of the change in adipose tissue. Both clinical and surgical approaches are described. Table 1 summarizes the approaches to each alteration.

\section{Fat atrophy ART switch}

Antiretroviral substitution ("switch") is a reasonable strategy to slow, stop, or even reverse the progression of lipoatrophy. Several studies have suggested switching thymidine analog NRTIs (d4T and ZDV) to alternative agents (abacavir or tenofovir). ${ }^{44}$ Gain in subcutaneous fat has been modest with this approach. ${ }^{44}$

Nucleoside-sparing regimes, as seen in ACTG 5142, are another option. The incidence of lipodystrophy was significantly higher in the efavirenz plus two NRTIs and lopinavir plus two NRTIs groups than in the efavirenz plus lopinavir group (32\% versus $17 \%$ versus $9 \%$, respectively). ${ }^{14}$ Therefore, one of our preferred approaches for treatment and/ or prevention is to change ART regimen.

A pilot study conducted in a reference center in Sao Paulo has evaluated a short-term treatment interruption strategy. Ten patients were treated for 7 days followed by 7 days of treatment interruption for 48 weeks among HIV-1-infected individuals with evidence of lipoatrophy. This small study demonstrated that lipoatrophy can be reduced with a $50 \%$ decrease in antiretroviral exposure over a 48 -week period without reducing the benefits of the antiretroviral treatment. Although low-level viremia was detectable in $15.8 \%$ of the monthly measurements, there were no repercussions in respect of the ability to fully suppress viremia thereafter. ${ }^{47}$

Table I Clinical diagnosis and treatment options common in Brazil for lipoatrophy and lipohypertrophy

\begin{tabular}{|c|c|c|}
\hline & Clinical diagnosis & Treatment \\
\hline Lipoatrophy & $\begin{array}{l}\text { Loss of subcutaneous fat } \\
\text { in face, limbs, buttocks } \\
\text { Musculature and veins } \\
\text { prominent }\end{array}$ & $\begin{array}{l}\text { Switch from stavudine } \\
\text { or zidovudine to } \\
\text { abacavir or tenofovir } \\
\text { Reconstructive } \\
\text { procedures }\end{array}$ \\
\hline Lipohypertrophy & $\begin{array}{l}\text { Measurements of weight, } \\
\text { height, body mass index, } \\
\text { abdominal circumference, }{ }^{a} \\
\text { and waist/hip ratio }\end{array}$ & $\begin{array}{l}\text { Diet/exercise } \\
\text { Metformin } \\
\text { Liposuction }\end{array}$ \\
\hline
\end{tabular}

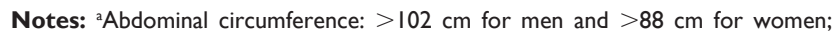
${ }^{\mathrm{b}}$ waist/hip ratio: $>0.95$ in men and $>0.85$ in women.
However, the current standard of care in ART does not indicate such a strategy to manage HIV patients. The Strategies for Management of Antiretroviral Therapy (SMART) study clearly demonstrated that stopping antiretrovirals can increase the risk of death and cardiovascular events, making this an unsuitable alternative. ${ }^{48}$

\section{Surgery}

Surgical treatment for lipoatrophy includes plastic surgery and gel fillers. Brazil was the first country where the government provided surgical treatment to HIV-associated lipodystrophy. Patients who fulfil criteria are referred to specialized centers. Depending on the situation, treatment includes gel fillers (polymethylmethacrylate) to facial lipoatrophy; liposuction of dorsocervical, abdominal, or dorsal fat; breast enlargement; and buttock implants. ${ }^{42}$

Injectable fillers can be permanent or temporary. Permanent fillers (polyalkylamide and polymethylmethacrylate) are synthetic materials that permanently fill in the space caused by loss of facial fat. Permanent fillers are not approved by the US Food and Drug Administration (FDA) and can possibly be associated with more implant-associated complications. ${ }^{49,50}$ Temporary fillers are biodegradable and immunologically inert material but have to be reinjected several times. ${ }^{49,51}$ A Brazilian cohort of 49 patients treated for lipoatrophy with polymethylmethacrylate showed no adverse effects in $76 \%$ of the patients. ${ }^{52}$

\section{Other approaches}

Thiazolidinedione drugs are of possible benefit in the treatment of lipoatrophy, but their use remains investigational. Rosiglitazone and pioglitazone are insulin-sensitizing agents used in the treatment of diabetes, and both were studied in HIV-associated lipodystrophy. Rosiglitazone does not show any increase in limb fat in some studies (although patients remained using $\mathrm{d} 4 \mathrm{~T}) ;^{53,54}$ however, it was associated with peripheral fat gain in patients with insulin resistance. ${ }^{55}$ Pioglitazone was evaluated in one placebo-controlled trial of 130 individuals, and a substantial increase in limb fat has been described in patients not using d4T. ${ }^{56}$ Rosiglitazone appears to increase the risk of myocardial infarction in diabetics, although the significance of these findings remains uncertain in nondiabetic patients. ${ }^{57}$ There are no studies in Brazil evaluating the use of thiazolidinediones.

Other medical options, such as uridine and leptin supplementation, have been studied in small trials, ${ }^{12}$ but there is no register of their use in Brazil. The most frequent clinical approach in Brazil is switching antiretrovirals. 


\section{Fat accumulation}

Potential approaches for fat deposition include exercise, medical therapy, and surgical interventions. Diet and exercise are a safe and potentially useful intervention for patients with abdominal fat accumulation, although efficacy data are limited. Weight reduction may be accompanied by some peripheral fat loss as well as central fat loss. Studies of HIVpositive people demonstrate that endurance and resistance exercise can reduce visceral adipose tissue. ${ }^{58,59}$

A randomized controlled study conducted in Brazil evaluated the impact of diet and aerobic training in 70 HIV-positive individuals. The intervention group received supervised aerobic training three times a week and monthly dietary counseling by a nutrition specialist, while the control group received just counseling sessions. After 24 weeks, improvement in all variables was observed, but the only significant changes were in muscle mass, hip circumference, and resting heart rate. Although this trial was not designed to evaluate specifically the impact on lipohypertrophy, anthropometrics parameters improved in all patients submitted to a simple intervention. ${ }^{60}$

The low cost of such an intervention is an attractive point favoring its use in a public health setting, especially in Brazil, where the government provides antiretroviral treatment. However, different professionals are involved in this type of intervention and they cannot always be available in all public HIV clinics of the country.

Different from fat atrophy, there is not sufficient evidence to recommend switching strategies in the treatment of fat deposition. Studies have failed to demonstrate a decrease in visceral fat. ${ }^{61}$ However, at least one study showed a clear improvement in visceral fat accumulation and insulin sensitivity following switching from lopinavir/ritonavir to atazanavir/ritonavir. ${ }^{62}$ These changes also had a significant impact on serum levels of glucose and lipids, which significantly decreased after switching PI.

Recombinant human growth hormone (rhGH) is lypolitic. Some studies have evaluated the efficacy of rhGH in the treatment of patients with fat accumulation. Two controlled trials have demonstrated a decrease in visceral fat. Two unsolved questions have been raised after these studies: the optimal strategy for maintaining visceral fat reduction achieved with rhGH and how to deal with the possibility of glucose intolerance and diabetes as major adverse effects. ${ }^{63,64}$

Tesamorelin, an analog growth hormone-releasing hormone, is produced in the hypothalamus and stimulates growth hormone secretion by the pituitary gland. Growth hormone induces hepatic synthesis of insulin-like growth factor 1, which mediates most of the peripheral actions of growth hormone and seems not to be related to glucose intolerance. ${ }^{65,66}$

Two randomized trials evaluated the effect of tesamorelin on HIV-associated fat accumulation. In both studies, a $15 \%-18 \%$ decrease in visceral fat tissue was observed. ${ }^{65,66}$ Based on these data, the FDA approved tesamorelin for the treatment of abdominal obesity in HIV-infected patients in November 2010. However, the optimal use of tesamorelin has to be defined, especially due to the rapid reaccumulation of fat after treatment discontinuation and the lack of long-term safety data. There are no data about the use of tesamorelin in Brazil, even in reference centers.

Another noninvasive possibility is the use of another antidiabetic medication, metformin. Its use was evaluated in small trials and was associated with a reduction in visceral and subcutaneous fat in patients with hyperinsulinemia with exercise training. It is not recommended for routine use in nondiabetic patients. ${ }^{21,67}$

Surgical treatment includes liposuction or surgical removal of adipose tissue. As mentioned before, the Brazilian government provides liposuction of dorsocervical and reduction mammoplasty. ${ }^{38}$ Sometimes, reaccumulation of fat can occur after the surgery. ${ }^{21}$

Another approach to treat lipodystrophy is the use of testosterone. One ACTG study (ACTG 5079) demonstrated a significant reduction in abdominal fat after 24 weeks of therapy with testosterone. However, a similar decrease of appendicular fat reduction was also noted, which could make it difficult to treat patients with both central fat accumulation and appendicular fat atrophy ${ }^{68}$ In other studies, patients with lipodystrophy treated with leptin had a significant decrease in total visceral fat, without any impact on appendicular fat tissue. ${ }^{69,70}$

The most frequent approach to fat accumulation in Brazil is diet and exercise. A recent literature review demonstrated that benefits of exercise on lipodystrophy are associated with the type of exercise. Although aerobic practices are associated with a positive impact on weight, decreased waist/hip ratio, and aerobic capacity, endurance is associated with an increase in lean mass, muscle mass, strength, and mineral bone density. The combination of both seems to be the best option, with a positive impact on all parameters. ${ }^{71} \mathrm{~A}$ switching strategy has not been used routinely. In severe cases, a surgical approach is a reasonable option, although it will not correct changes in metabolism.

\section{Conclusion}

The ARV drugs largely used during the early HAART era were significantly associated with some important metabolic 
effects, with a great potential to significantly decrease the quality of life of HIV patients and the ability to impair some of the gains resulting from HAART. The development of newer, friendlier, and safer ARV drugs minimized these problems for patients initiating therapy in the late HAART era. However, a large number of individuals who were exposed to older drugs in the past were severely affected by these body changes, with a significant impact on their self-esteem and quality of life.

Several trials have tried to find a way to treat these body changes, but no single solution has been found so far. Potential treatment approaches including changing lifestyle, with adherence to adequate nutritional habits, physical exercise, and some medical interventions, demonstrated modest net benefits. In some extreme cases, surgical interventions are needed in order to mitigate the consequences of fat deposition/atrophy. However, there is still an unmet need for better treatments for lipodystrophy. Starting ARV therapy nowadays is much easier than in the past, with at least 28 licensed ARV drugs. Moreover, most of the recent available ARV drugs are safer than those used in the early HAART era, making prevention of lipodystrophy a main strategy in the management of this serious health problem. Also, it would be desirable to have easier access to these newer medications to middle and low-income countries, meaning lowering costs in order to try to minimize these body modifications related to older HAART regimens. This will ultimately improve antiretroviral efficacy while improving not only survival but also quality of life of HIVinfected individuals.

\section{Disclosure}

The authors report no conflicts of interest in this work.

\section{References}

1. Palella FJ Jr, Delaney KM, Moorman AC; for HIV Outpatient Study Investigators. Declining morbidity and mortality among patients with advanced human immunodeficiency virus infection. $N$ Engl J Med. 1998;338:853-860.

2. Tuboi SH, Brinkhof MWG, Egger M, et al. Discordant responses to potent antiretroviral treatment in previously naive HIV-1-infected adults initiating treatment in resource-constrained countries: the antiretroviral therapy in low-income countries (ART-LINC) collaboration. J Acquir Immune Defic Syndr. 2007;45:52-59.

3. Marins JR, Jamal LF, Chen SY, et al. Dramatic improvement in survival among adult Brazilian AIDS patients. AIDS. 2003;17:1675-1682.

4. Ministry of Health - Bureau of Health Surveillance - Department of STD, AIDS and Viral Hepatitis. Boletim Epidemiológico - Aids e DST Ano II - No 1 [Epidemiological Bulletin - Aids and STDs Year II No 1.- Brazil 2013. Portugese.

5. Lohse N, Hansen AB, Gerstoft J, Obel N. Improved survival in HIV-infected persons: consequences and perspectives. J Antimicrob Chemother. 2007;60:461-463.
6. Carr A, Samaras K, Burton S, et al. A syndrome of peripheral lipodystrophy, hyperlipidaemia and insulin resistance in patients receiving HIV protease inhibitors. AIDS. 1998;12:F51.

7. Tien PC, Cole SR, Williams CM, et al. Incidence of lipoatrophy and lipohypertrophy in the women's interagency HIV study. JAcquir Immune Defic Syndr. 2003;34(5):461-466.

8. Mulligan K, Parker RA, Komarow L, et al. Mixed patterns of changes in central and peripheral fat following initiation of antiretroviral therapy in a randomized trial. J Acquir Immune Defic Syndr. 2006;41(5): 590-597.

9. Santos CP, Felipe YX, Braga PE, Lima RO, Segurado AC. Self-perception of body changes in persons living with HIV/ AIDS: prevalence and associated factors. AIDS. 2005;19 Suppl 4: S14-S21.

10. Monnerat BZ, Cerutti Junior C, Caniçali SC, Motta TR. Clinical and biochemical evaluation of HIV-related lipodystrophy in an ambulatory population from the Hospital Universitário Cassiano Antonio de Morais, Vitória, ES, Brazil. Braz J Infect Dis. 2008;12(4):364-368.

11. Sprinz E, Lazzaretti RK, Kuhmmer R, Ribeiro JP. Dyslipidemia in HIV-infected individuals. Braz J Infect Dis. 2010;14(6):575-588.

12. Joly V, Flandre P, Meiffredy V, et al. Increased risk of lipoatrophy under stavudine in HIV-1 infected patients: results of a substudy from a comparative trial. AIDS. 2002;16:2447-2457.

13. de Waal R, Cohen K, Maartens G. Systematic review of antiretroviralassociated lipodystrophy: lipoatrophy, but not central fat gain, is an antiretroviral adverse drug reaction. PLoS One. 2013;8(5):e63623.

14. Haubrich RH, Riddler SA, DiRienzo AG, et al. Metabolic outcomes in a randomized trial of nucleoside, nonnucleoside and protease inhibitor-sparing regimens for initial HIV treatment. AIDS. 2009;23:1109.

15. Sax PE, Kumar P. Tolerability and safety of HIV protease inhibitors in adults. J Acquir Immune Defic Syndr. 2004;37(1):1111-1124.

16. Brown TT, Chu H, Wang Z, Palella FJ, et al. Longitudinal increases in waist circumference are associated with HIV-serostatus, independent of antiretroviral therapy. AIDS. 2007;21(13):1731-1738.

17. Kotler DP. HIV lipodystrophy etiology and pathogenesis. Body composition and metabolic alterations: etiology and pathogenesis. AIDS Read. 2003;13 Suppl 4:S5-S9.

18. Justman JE, Hoover DR, Shi Q, Tan T, et al. Longitudinal anthropometric patterns among HIV-infected and HIV-uninfected women. J Acquir Immune Defic Syndr. 2008;47(3):312-319.

19. Bacchetti P, Gripshover B, Grunfeld C, et al. Fat distribution in men with HIV infection. J Acquir Immune Defic Syndr. 2005;40:121.

20. Study of Fat Redistribution and Metabolic Change in HIV Infection (FRAM). Fat distribution in women with HIV infection. J Acquir Immune Defic Syndr. 2006;42:562.

21. Wohl DA, McComsey G, Tebas P, et al. Current concepts in the diagnosis and management of metabolic complications of HIV infection and its therapy. Clin Infect Dis 2006;43:645-653.

22. Ofotokun I, Ribaudo H, Na L, et al. Darunavir or atazanavir vs raltegravir lipid changes are unlinked to ritonavir exposure: ACTG 5257. Proceedings of the Conference on Retroviruses and Opportunistic Infections. March 3-6, 2014. Boston, MA. Abstract 746.

23. Galescu O, Bhangoo A, Ten S. Insulin resistance, lipodystrophy and cardiometabolic syndrome in HIV/AIDS. Rev Endocr Metab Disord. 2013;14(2):133-140.

24. Lazzaretti RK, Gasparotto AS, Sassi MG, et al. Genetic markers associated to dyslipidemia in HIV-infected individuals on HAART. ScientificWorldJournal. 2013;2013:608415. eCollection 2013.

25. Shikuma CM, Yang Y, Glesby MJ, et al. Metabolic effects of protease inhibitor-sparing antiretroviral regimens given as initial treatment of HIV-1 Infection (AIDS Clinical Trials Group Study A5095). J Acquir Immune Defic Syndr. 2007;44:540.

26. Guaraldi G1, Luzi K, Bellistrì GM, et al. CD8 T-cell activation is associated with lipodystrophy and visceral fat accumulation in antiretroviral therapy-treated virologically suppressed HIV-infected patients. J Acquir Immune Defic Syndr. 2013;64(4):360-366. 
27. Mulligan K, Anastos K, Justman J, et al. Fat distribution in HIV-infected women in the United States: DEXA substudy in the Women's Interagency HIV Study. J Acquir Immune Defic Syndr. 2005;38:18-22.

28. Jacobson DL, Knox T, Spiegelman D, Gorbach S, Wanke C. Prevalence of, evolution of, and risk factors for fat atrophy and fat deposition in a cohort of HIV-infected men and women. Clin Infect Dis. 2005;40(12): 1837-1845.

29. Hendricks KM, Dong KR, Tang AM, et al. High-fiber diet in HIV-positive men is associated with lower risk of developing fat deposition. Am J Clin Nutr. 2003;78:790.

30. Currier JS, Lundgren JD, Carr A, et al. Epidemiological evidence for cardiovascular disease in HIV-infected patients and relationship to highly active antiretroviral therapy. Circulation. 2008;118(2): e29-e35.

31. Currier JS, Taylor A, Boyd F, et al. Coronary heart disease in HIV-infected individuals. J Acquir Immune Defic Syndr. 2003;33:506-512.

32. Friis-Møller N, Sabin CA, Weber R, et al. Data Collection on Adverse Events of Anti-HIV Drugs (DAD) Study Group. Combination antiretroviral therapy and the risk of myocardial infarction [published correction appears in $N$ Engl J Med. 2004;350:955]. $N$ Engl J Med. 2003;349:1993-2003.

33. Friis-Møller N, Reiss P, Sabin CA, et al; DAD Study Group. Class of antiretroviral drugs and the risk of myocardial infarction. $N$ Engl J Med. 2007;356:1723-1735.

34. Carr A, Emery S, Law M; for HIV Lipodystrophy Case Definition Study Group. An objective case definition of lipodystrophy in HIV-infected adults: a case-control study. Lancet. 2003;361(9359):726-735.

35. Heath KV, Hogg RS, Chan KJ, et al. Lipodystrophy-associated morphological, cholesterol and triglyceride abnormalities in a population-based HIV/AIDS treatment database. AIDS. 2001;15(2):231-239.

36. Leitz G, Robinson P. The development of lipodystrophy on a protease inhibitor-sparing highly active antiretroviral therapy regimen. AIDS 2000;14(4):468-469.

37. Mallon PW, Miller J, Cooper DA, Carr A. Prospective evaluation of the effects of antiretroviral therapy on body composition in HIV-1-infected men starting therapy. AIDS. 2003;17(7):971-979.

38. Palella FJ Jr, Cole SR, Chmiel JS, et al. Anthropometrics and examinerreported body habitus abnormalities in the multicenter AIDS cohort study. Clin Infect Dis. 2004;38(6):903-907.

39. Ceccato MG, Bonolo PF, Souza Neto AI, Araújo FS, Freitas MI Antiretroviral therapy-associated dyslipidemia in patients from a reference center in Brazil. Braz J Med Biol Res. 2011;44(11): 1177-1183.

40. Kroll AF, Sprinz E, Leal SC, Labrêa Mda G, Setúbal S. Prevalence of obesity and cardiovascular risk in patients with HIV/AIDS in Porto Alegre, Brazil. Arq Bras Endocrinol Metabol. 2012;56(2): 137-141.

41. Lazzaretti RK, Kuhmmer R, Sprinz E, Polanczyk CA, Ribeiro JP Dietary intervention prevents dyslipidemia associated with highly active antiretroviral therapy in human immunodeficiency virus type 1-infected individuals: a randomized trial. J Am Coll Cardiol. 2012;59(11):979-988.

42. Síndrome Lipodistrófica no HIV. [Lipodystrophy syndrome in HIV] Brazil. Ministry of Health. 2011. Available from http://www.aids.gov.br/ sites/default/files/anexos/publicacao/2011/50427/vers_o_final_63134. pdf. Accessed July 9, 2014.

43. Anjos EM, Pfrimer K, Machado AA, Cunha SF, Salomão RG, Monteiro JP. Nutritional and metabolic status of HIV-positive patients with lipodystrophy during one year of follow-up. Clinics (Sao Paulo). 2011;66(3):407-410.

44. Carr A, Workman C, Smith DE, et al. Mitochondrial Toxicity (MITOX) Study Group. Abacavir substitution for nucleoside analogs in patients with HIV lipoatrophy: a randomized trial. JAMA. 2002;288(2): 207-215.

45. Moyle GJ, Sabin CA, Cartledge J, et al. A randomized comparative trial of tenofovir DF or abacavir as replacement for a thymidine analogue in persons with lipoatrophy. AIDS. 2006;20(16):2043-2050.
46. Martin A, Smith DE, Carr A, et al. Reversibility of lipoatrophy in HIV-infected patients 2 years after switching from a thymidine analogue to abacavir: the MITOX Extension Study. AIDS. 2004;18:1029.

47. Gosuen GC, Turcato G Jr, Salomao R, Lewi DS, Diaz RS. Decrease in lipoatrophy in a pilot study using a short-term treatment interruption strategy for 48 weeks in São Paulo, Brazil. AIDS Res Hum Retroviruses. 2012;28(8):747-748.

48. El-Sadr WM, Lundgren J, Neaton JD; for Strategies for Management of Antiretroviral Therapy (SMART) Study Group. CD4+ count-guided interruption of antiretroviral treatment. $N$ Engl J Med. 2006;355(22): 2283-2296.

49. Sutinen J. Interventions for managing antiretroviral therapy-associated lipoatrophy. Curr Opin Infect Dis. 2005;18(1):25-33.

50. Nadarajah JT, Collins M, Raboud J, et al. Infectious complications of Bio-Alcamid filler used for HIV-related facial lipoatrophy. Clin Infect Dis. 2012;55(11):1568-1574.

51. Valantin MA, Aubron-Olivier C, Ghosn J, et al. Polylactic acid implants (New-Fill) to correct facial lipoatrophy in HIV-infected patients: results of the open-label study VEGA. AIDS. 2003;17(17):2471-2477.

52. Orsi AT, Miranda AE, Souza AC, et al. Lipoatrophy in patients with AIDS: treatment with polymethylmethacrylate in Amazonas, Brazil. Int J Dermatol. 2011;50(10):1255-1258.

53. Carr A, Workman C, Carey D, et al. No effect of rosiglitazone for treatment of HIV-1 lipoatrophy: randomised, double-blind, placebo-controlled trial. Lancet. 2004;363:429.

54. Hadigan C, Yawetz S, Thomas A, et al. Metabolic effects of rosiglitazone in HIV lipodystrophy: a randomized, controlled trial. Ann Intern Med. 2004; 140:786.

55. Tungsiripat M, Bejjani DE, Rizk N, et al. Rosiglitazone improves lipoatrophy in patients receiving thymidine-sparing regimens. AIDS. 2010;24:1291

56. Slama L, Lanoy E, Valantin MA, et al. Effect of pioglitazone on HIV1-related lipodystrophy: a randomized double-blind placebo-controlled trial (ANRS 113). Antivir Ther. 2008;13:67.

57. Nissen SE, Wolski K. Effect of rosiglitazone on the risk of myocardial infarction and death from cardiovascular causes. $N$ Engl J Med. 2007;356:2457.

58. Roubenoff R, Weiss L, McDermott A, Heflin T, Cloutier GJ, Wood M, et al. A pilot study of exercise training to reduce trunk fat in adults with HIV-associated fat redistribution. AIDS. 1999;13(11):1373-1375.

59. Thöni GJ, Fedou C, Brun JF, et al. Reduction of fat accumulation and lipid disorders by individualized light aerobic training in human immunodeficiency virus infected patients with lipodystrophy and/or dyslipidemia. Diabetes Metab. 2002;28(5):397-404.

60. Ogalha C, Luz E, Sampaio E, et al. A randomized, clinical trial to evaluate the impact of regular physical activity on the quality of life, body morphology and metabolic parameters of patients with AIDS in Salvador, Brazil. J Acquir Immune Defic Syndr. 2011;57 Suppl 3:S179-S185.

61. Drechsler H1, Powderly WG. Switching effective antiretroviral therapy: a review. Clin Infect Dis. 2002;35(10):1219-1230.

62. Stanley TL, Joy T, Hadigan CM, et al. Effects of switching from lopinavir/ritonavir to atazanavir/ritonavir on muscle glucose uptake and visceral fat in HIV-infected patients. AIDS. 2009;23(11):1349-1357.

63. Grunfeld C, Thompson M, Brown SJ, et al. Recombinant human growth hormone to treat HIV-associated adipose redistribution syndrome: 12 week induction and 24-week maintenance therapy. JAcquir Immune Defic Syndr. 2007;45:286.

64. Lo J, You SM, Canavan B, et al. Low-dose physiological growth hormone in patients with HIV and abdominal fat accumulation: a randomized controlled trial. JAMA. 2008;300:509.

65. Falutz J, Potvin D, Mamputu JC, et al. Effects of tesamorelin, a growth hormone-releasing factor, in HIV-infected patients with abdominal fat accumulation: a randomized placebo-controlled trial with a safety extension. J Acquir Immune Defic Syndr. 2010;53(3): 311-322.

66. Falutz J, Allas S, Blot K, et al. Metabolic effects of a growth hormonereleasing factor in patients with HIV. N Engl J Med. 2007;357:2359. 
67. Hadigan C, Corcoran C, Basgoz N, Davis B, Sax P, Grinspoon S. Metformin in the treatment of HIV lipodystrophy syndrome: a randomized controlled trial. JAMA. 2000;284(4):472-477.

68. Bhasin S, Parker RA, Sattler F; for AIDS Clinical Trials Group Protocol A5079 Study Team. Effects of testosterone supplementation on whole body and regional fat mass and distribution in human immunodeficiency virus-infected men with abdominal obesity. J Clin Endocrinol Metab. 2007;92(3):1049-1057.

69. Mulligan K, Khatami H, Schwarz JM, et al. Improvements in hepatic and adipocyte insulin sensitivity, dyslipidemia, and visceral fat during leptin treatment in HIV-infected men with lipoatrophy and hypoleptinemia. Proceedings of the Conference on Retroviruses and Opportunistic Infections. February 25-28, 2007; Los Angeles, CA. Abstract 805.
70. Nagy GS, Tsiodras S, Martin LD, et al. Human immunodeficiency virus type 1-related lipoatrophy and lipohypertrophy are associated with serum concentrations of leptin. Clin Infect Dis. 2003;36(6):795-802.

71. Gomes-Neto M, Conceição CS, Oliveira Carvalho V, Brites C. A systematic review of the effects of different types of therapeutic exercise on physiologic and functional measurements in patients with HIV/AIDS. Clinics (Sao Paulo). 2013;68(8):1157-1167.

\section{Publish your work in this journal}

Therapeutics and Clinical Risk Management is an international, peerreviewed journal of clinical therapeutics and risk management, focusing on concise rapid reporting of clinical studies in all therapeutic areas, outcomes, safety, and programs for the effective, safe, and sustained use of medicines. This journal is indexed on PubMed Central, CAS,
EMBase, Scopus and the Elsevier Bibliographic databases. The manuscript management system is completely online and includes a very quick and fair peer-review system, which is all easy to use. Visit http://www.dovepress.com/testimonials.php to read real quotes from published authors.

Submit your manuscript here: http://www.dovepress.com/therapeutics-and-clinical-risk-management-journal 